\title{
Case report of recurrent fibromatosis with laryngeal involvement: Treatment based on network analyses of NGS data
}

\author{
NATHAN LLOYD ${ }^{1,2}$, JONATHAN KOPEL $^{3}$, SANJAY AWASTHI $^{3}$ and JOEHASSIN CORDERO ${ }^{1,2}$ \\ Departments of ${ }^{1}$ Surgery, ${ }^{2}$ Otolaryngology and ${ }^{3}$ Internal Medicine, Division of Heme/Oncology, \\ Texas Tech University Health Sciences Center, Lubbock, TX 79430, USA
}

Received July 26, 2021; Accepted September 30, 2021

DOI: $10.3892 / \mathrm{mco} .2022 .2506$

\begin{abstract}
Aggressive fibromatosis (AF) is a rare, benign neoplasm originating from musculoaponeurotic stromal structures characterized by aggressive growth and infiltration of local tissues. To date, only six previous cases of AF involving the larynx have been reported. The present case was that of a 70-year-old female patient with a 5-year history of hoarseness and an enlarging neck mass consistent with aggressive recurrent fibromatosis. MRI displayed a large, solid mass arising from the left anterior cervical space displacing the trachea and upper airway to the right. At one year after the initial radical resection, the patient presented with recurrence. A second radical excision was performed not including a laryngectomy. Radiation therapy was considered for possible local treatment to prevent subsequent tumor recurrence. According to the literature, AF has recurrence rates of up to $40-70 \%$ within 18 months. There is only sparse literature to guide treatment. Using the mutations detected in the patient's AF tissue, an Ingenuity Pathway Analysis (IPA) was used to guide treatment of the recurrence. In the present case, the IPA analysis indicated the use of pazopanib to treat the patient's cancer. In general, surgery appears to be the treatment of choice for head and neck AF, but the management of recurrence is controversial.
\end{abstract}

\section{Introduction}

Aggressive fibromatosis (AF) is a rare, benign neoplasm with an incidence of 2 to 4 per 1 million individuals annually. It originates from musculoaponeurotic stromal structures and aggressively grows and infiltrates local tissues, principally the connective tissue of the muscle and overlying fascia or

Correspondence to: Dr Jonathan Kopel, Department of Internal Medicine, Division of Heme/Oncology, Texas Tech University Health Sciences Center, 3,601 Fourth Street Stop 6,238, Lubbock, TX 79430, USA

E-mail: jonathan.kopel@ttuhsc.edu

Key words: aggressive fibromatosis, surgery, chemotherapy, management aponeurosis (1). More commonly, AF occurs in the head and neck region, followed by the face, oral cavity, scalp, paranasal sinus and orbit. Specifically, $10 \%$ of reported AF cases appear in the cervical region and only six cases have reported involvement of the larynx (2). Specifically, AF of the head and neck region tends to be more locally aggressive, making complete resection difficult (3). As a result, AF has a high recurrence rate despite successful surgical resection. Therefore, there is of interest whether chemoadjuvant therapies may reduce cancer resection after surgical resection. The current study presented a case of aggressive recurrent fibromatosis involving the left anterior cervical neck along with the thyroid in a 70 -year-old female who underwent multiple neck resections.

\section{Case report}

A 70-year-old female patient (body mass index, $23.6 \mathrm{~kg} / \mathrm{m}^{2}$ ) presented at the Otolaryngology Department Clinic at Texas Tech University Health Science Center (Lubbock, USA) with an approximately three-to-four-year history of an enlarging left neck mass. The patient did not seek any treatment previously because she was afraid of the possible diagnosis. She denied any pain, dysphagia or dyspnea. The patient's most noticeable symptom was a higher vocal pitch. The family history of the patient included pneumonia in the father, hypothyroidism in the patient's sister and prostate cancer in the patient's brother. On examination, an anterior displacement of the trachea at the C4-C5 level was detected. Upon further inspection, a fixed, firm $>10-\mathrm{cm}$ mass with a superior boundary to the inferior portion of the left mandible and an inferior boundary of the left thyroid cartilage was noted. Anterior and lateral X-ray of the neck revealed a soft tissue mass on the neck (Fig. 1). MRI indicated a large, solid mass measuring $10.8 \times 7.5 \times 9.7 \mathrm{~cm}$, which expanded from the left anterior cervical space displacing the trachea by $2-2.5 \mathrm{~cm}$ to the right (Figs. 2 and 3). There was no involvement of any other organs besides the larynx. Blood chemistry analysis was also significant for leukopenia. The mass was inferior to the parotid glands, invading medially and posteriorly to the trachea with medial and inferior extension to the left lobe of the thyroid. Histological analysis indicated an atypical and cellular spindle cell neoplasm with low proliferative activity exhibiting fascicular and storiform growth patterns. No necrosis was observed. A diagnosis of a low-grade myofibroblastic sarcoma was made with a pathological stage 
A

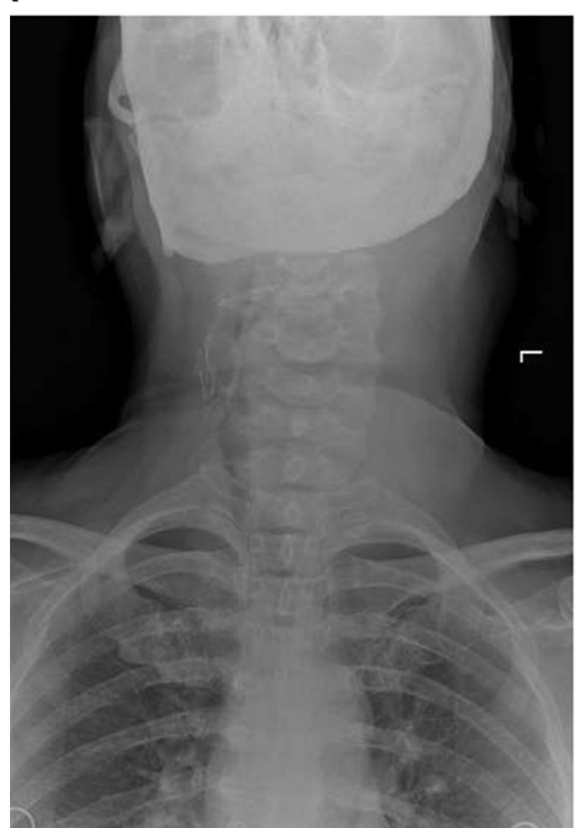

B

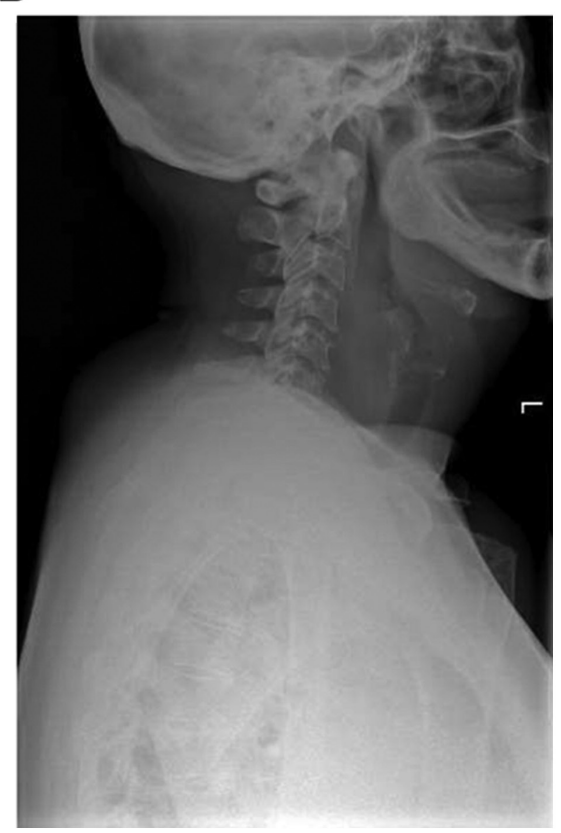

Figure 1. (A) Anterior and (B) lateral X-ray displaying soft tissue sarcoma of the neck.

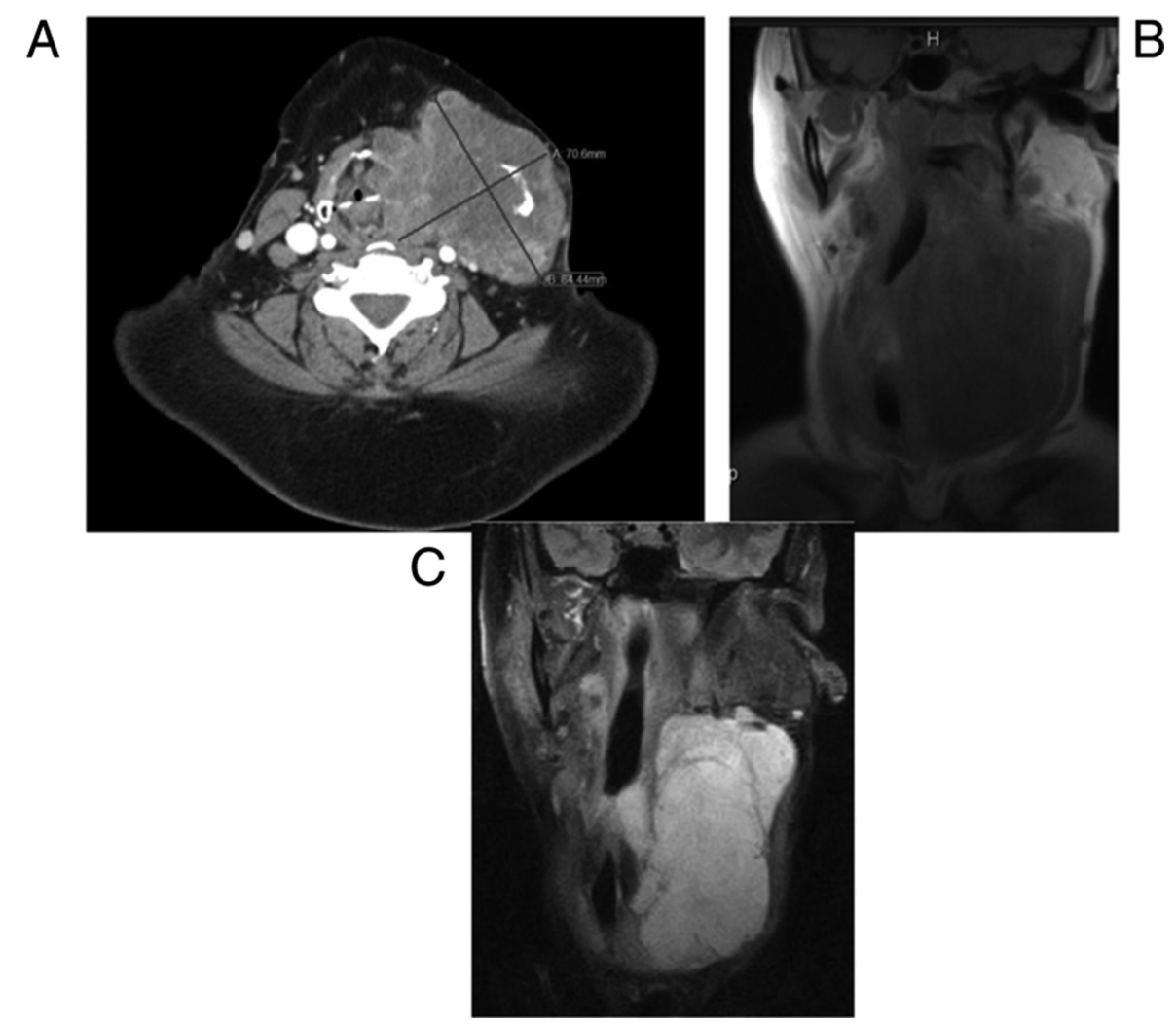

Figure 2. (A) T1-weighted non-contrast MRI demonstrating left neck mass with compression of midline structures. (B) Short tau inversion recovery MRI displaying left neck mass with compressive symptoms. (C) CT scan with contrast demonstrating recurrence of aggressive fibromatosis. The images were all acquired at the same time at the initial presentation of the patient (scale bar, $\mathrm{cm}$ ).

of rPT4aNxMx. Immunohistochemical analysis indicated that the tumor was smooth muscle actin-positive, SOX10-negative, desmin-negative, S100-negative, CD34-negative, pancytokeratin-negative and $\beta$-catenin-negative. Genetic analysis of the tumor suggested cyclin-dependent kinase 4 (CDK4) amplification, erb-b2 receptor tyrosine kinase (ERBB3) amplification, MDM2 amplification, colony stimulating factor 3 receptor (CSF3R) G21R mutation, FRS2 amplification, HMGA2-KERA fusion and RUNX family transcription factor 1 (RUNX1) partner transcriptional co-repressor 1 R373. 

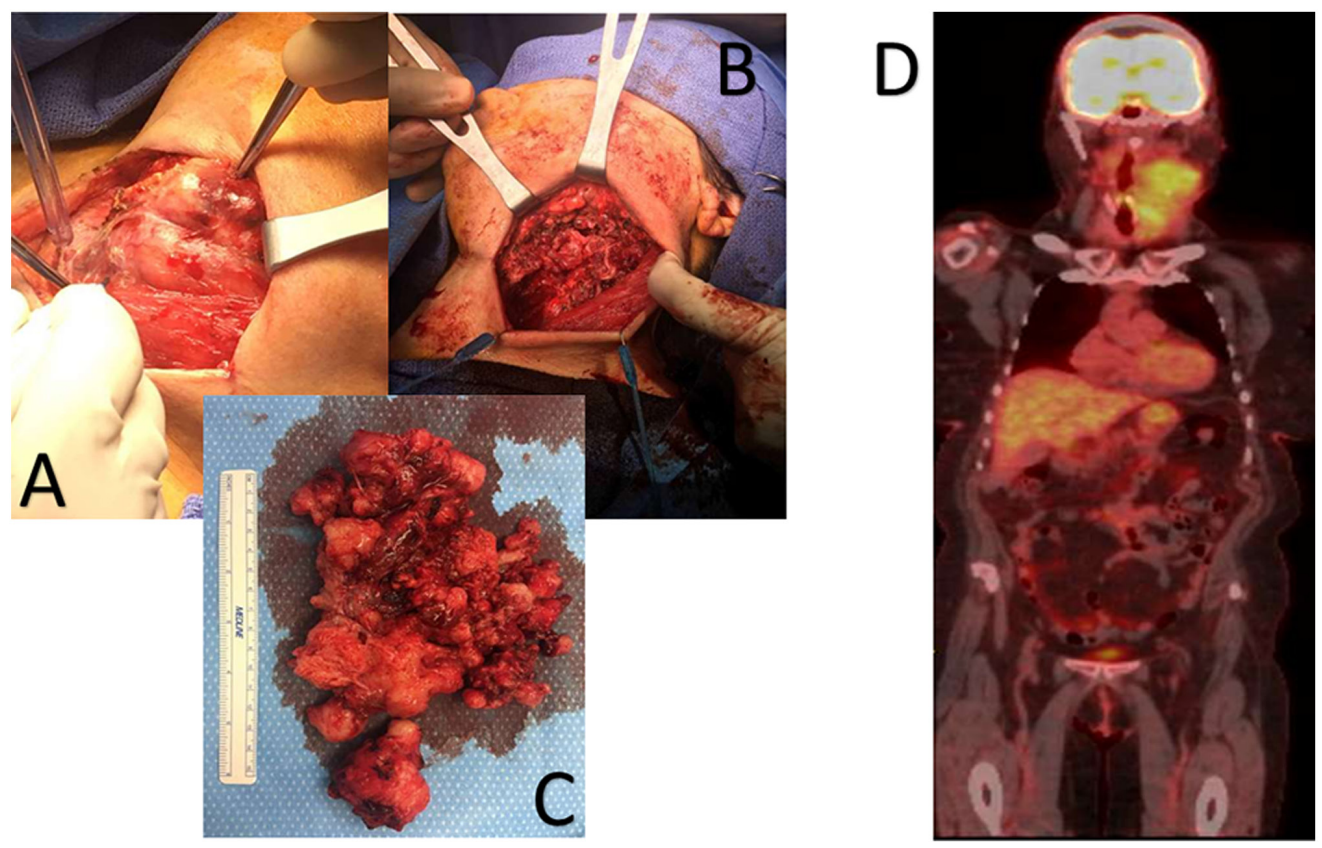

Figure 3. (A) Intraoperative image of the neck mass prior to resection; (B) intraoperative image of the neck after resection of the mass; (C) image of the resected mass; (D) positron emission tomography scan of aggressive angiofibromatosis. The images were all acquired at the same time during the removal of the tumor mass, apart from the PET scan, which was performed prior to resection of the original tumor.

An Ingenuity Pathway Analysis (IPA; Qiagen GmbH) was performed using the aforementioned genetic analysis data in the core IPA to develop an interactive network. The Qiagen IPA software (Qiagen $\mathrm{GmbH}$ ) was used to create the networks. The network was then overlaid with a network activity predictor to determine the effects of mutations on activating or de-activating proteins and/or transcriptions factors in the interactive network. The analysis suggested that these mutations affected numerous canonical pathways, including regulation of epithelial-mesenchymal growth factor pathways, Her-2 signaling, the BAG2 signaling pathway and the p14 tumor suppressor. The resulting IPA images are presented in Figs. 4-7.

The patient consented to surgery and the mass was excised by left modified neck dissection through levels I-VI with preservation of nerves, vessels and surrounding structures. The mass was carefully removed from the carotid sheath, strap muscles, anterior body of the thyroid and anterior trachea in succession. The mass was noted to extend into both retro-pharyngeal and retro-laryngeal spaces and was then removed posteriorly from the posterior trachea, thyroid cartilage and thyroid. The dissection was difficult due to aggressive local invasion; thus, clear margins were not achieved. The mass was at least $12-15 \mathrm{~cm}$ in length, $10 \mathrm{~cm}$ in width and $8 \mathrm{~cm}$ in height. It was sent for pathology, which indicated spindle cell proliferation consistent with deep fibromatosis with no clear margins. A post-operative CT scan with contrast was performed 8 months after surgery and indicated significant reduction of fibromatosis tissue in the left neck.

Another noteworthy finding included residual fibromatosis tissue between the lower cricoid cartilage and the medial aspect of the upper left thyroid lobe. At 15 months after resection, the patient returned to the clinic with a new chief complaint of left neck paresthesia with involvement of the left ear. The patient mentioned that she had not been able to sing since the operation. She confirmed hoarseness and dysphagia but denied any change in pitch and dyspnea. On physical exam, a noticeable mass of the left neck in the same location as the original mass was present. On follow-up 5 months later, the mass had roughly doubled in size. A CT with contrast indicated an $8.1 \times 8.5 \times 10.3 \mathrm{~cm}$ mass with a superior border to the mandibular ramus and inferior border of the left thyroid (Fig. 2). Invasion of the left para-pharyngeal space, rightward deviation of the airway and narrowing of the glottis was also noted on imaging. The mass invaded deeper up to the lateral edge of the tonsil. And into the left lateral margin of the tonsillar soft tissues and vallecula. The mass was resected $\sim 21$ months after the first dissection (Figs. 4-7). Left radical neck mass excision included levels II-IV, removing the mass from the left thyroid bed, left carotid sheath, left parotid bed and left posterior digastric muscle after careful mobilization of the sternocleidomastoid muscle. Erosion of the left thyroid cartilage into the pharynx was noted but the hyoid bone was left intact. After resection, radiation oncology was consulted for possible local radiation treatment to prevent tumor recurrence. A positron emission tomography scan using $15.97 \mathrm{mCi}$ of 18 -fluoro 2-deoxyglucose indicated a hypermetabolic mass extending inferiorly from the top of the left side of the thyroid and deviating and possibly invading the larynx, which is consistent with known primary malignancy (Fig. 8). The patient was subsequently given a total dose of 6,600 cGy of radiation in 33 fractions to her left neck and supraclavicular area. The patient developed a minor skin reaction and hoarseness in response to the radiation therapy but otherwise tolerated it well. After radiation therapy, remnants of the sarcoma still remained in the left neck. As presented in Figs. 4-7, an IPA analysis was performed to determine which chemotherapy regimens would be most effective at eliminating the sarcoma. The network suggested that pazopanib would be a possible chemotherapeutic agent against AF by inhibiting the 


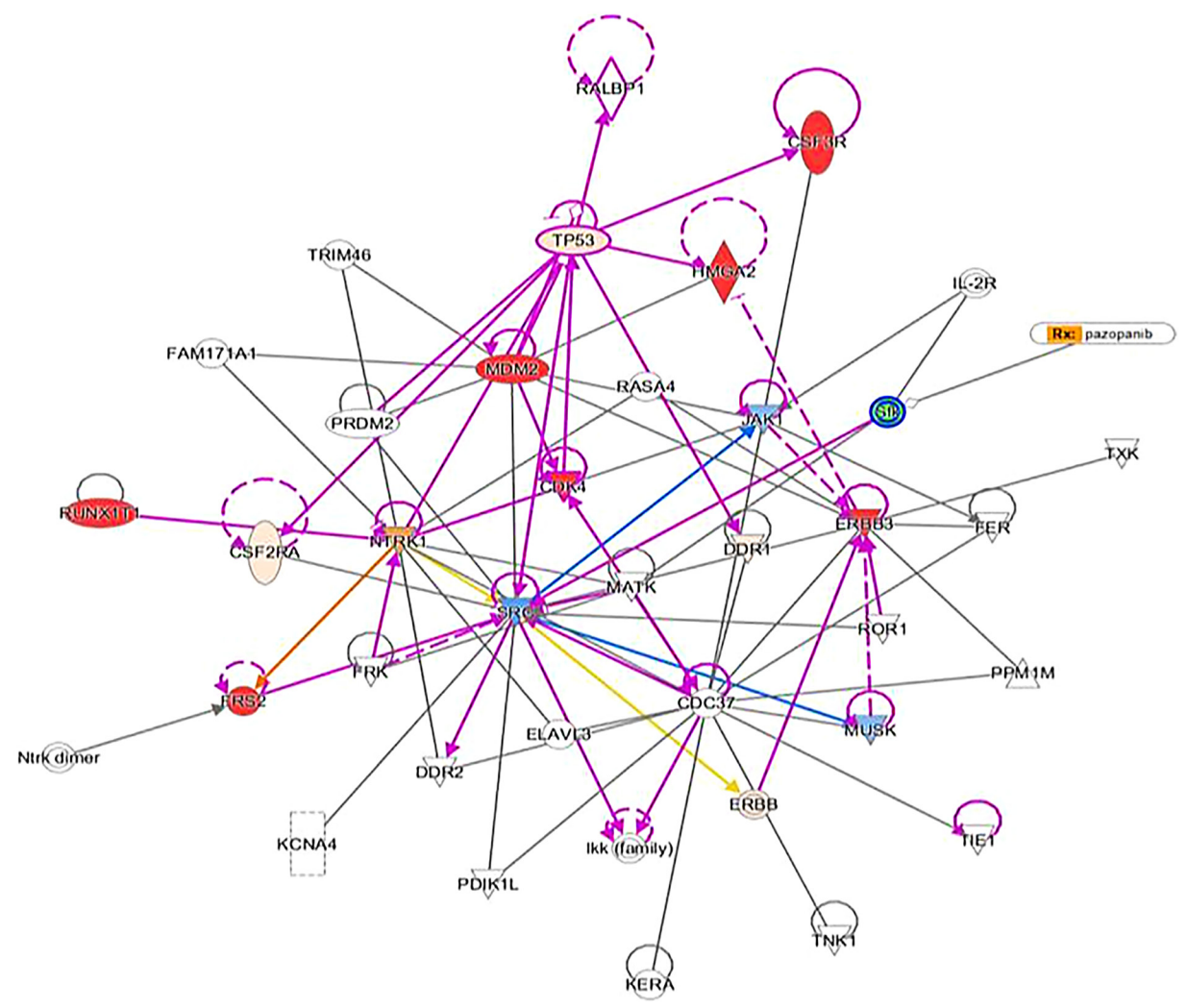

Figure 4. Ingenuity pathway analysis indicating pazopanib. The red geometric shape indicates an increase in measurement. The green geometric shape indicates a decrease in measurement. The orange geometric shape indicates an increase in prediction of the activity of connected signaling node. The blue geometric node indicates a decrease in prediction of the activity of connected signaling node. The glow around a geometric shape indicates activity when opposite of measurement. The orange line indicates an interaction leading to activation. The blue line indicates an interaction leading to inhibition. The yellow line indicates findings inconsistent with state of downstream molecule. The grey line indicates effect not predicted.

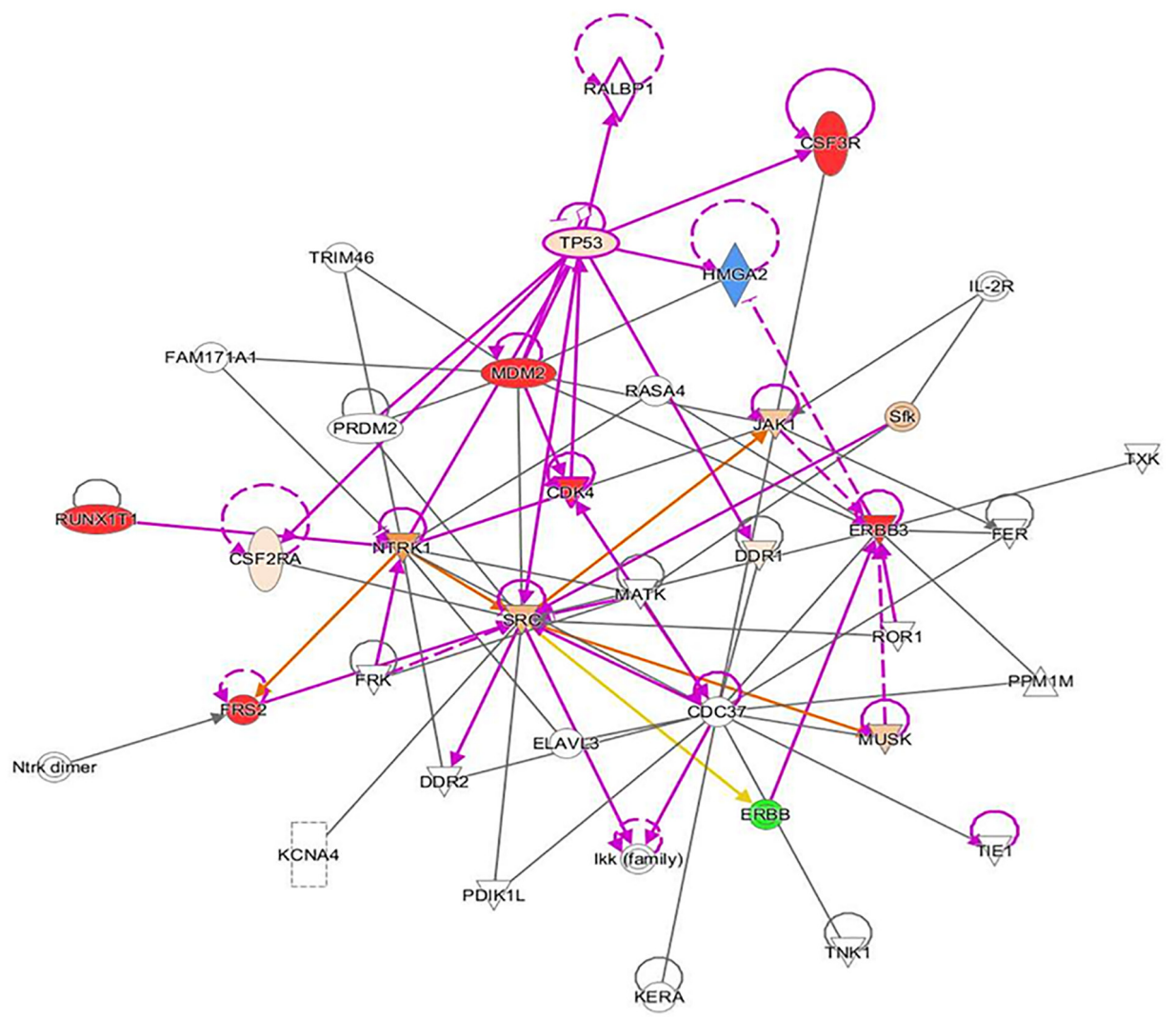

Figure 5. Ingenuity pathway analysis suggesting ERBB inhibitors. Refer to Figure 4 legend for explanation of figure contentss. ERBB, erb-b2 receptor tyrosine kinase. 


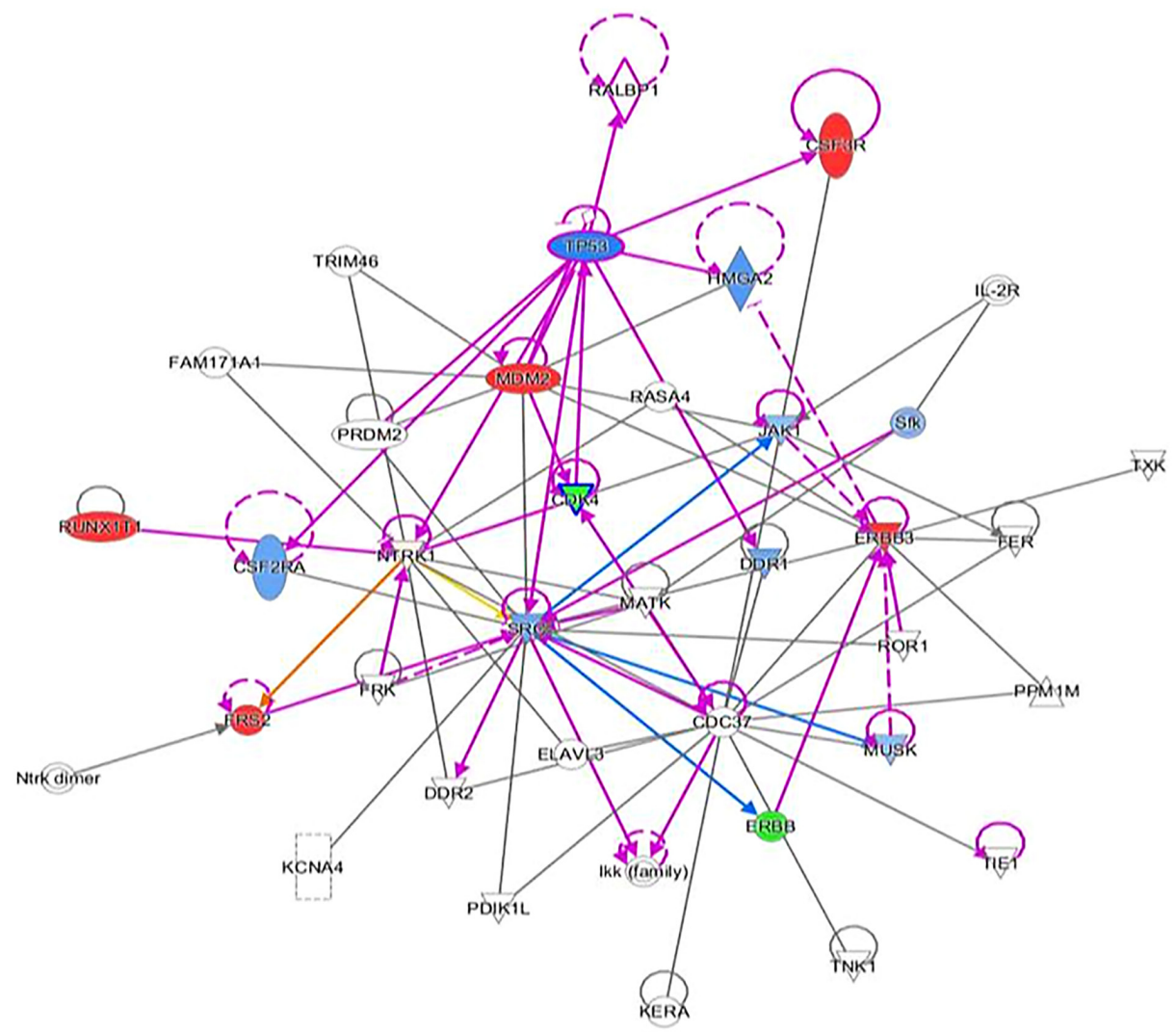

Figure 6. Ingenuity pathway analysis suggesting CDK4 inhibitors. Refer to Figure 4 legend for explanation of figure contents. CDK4, cyclin-dependent kinase 4.

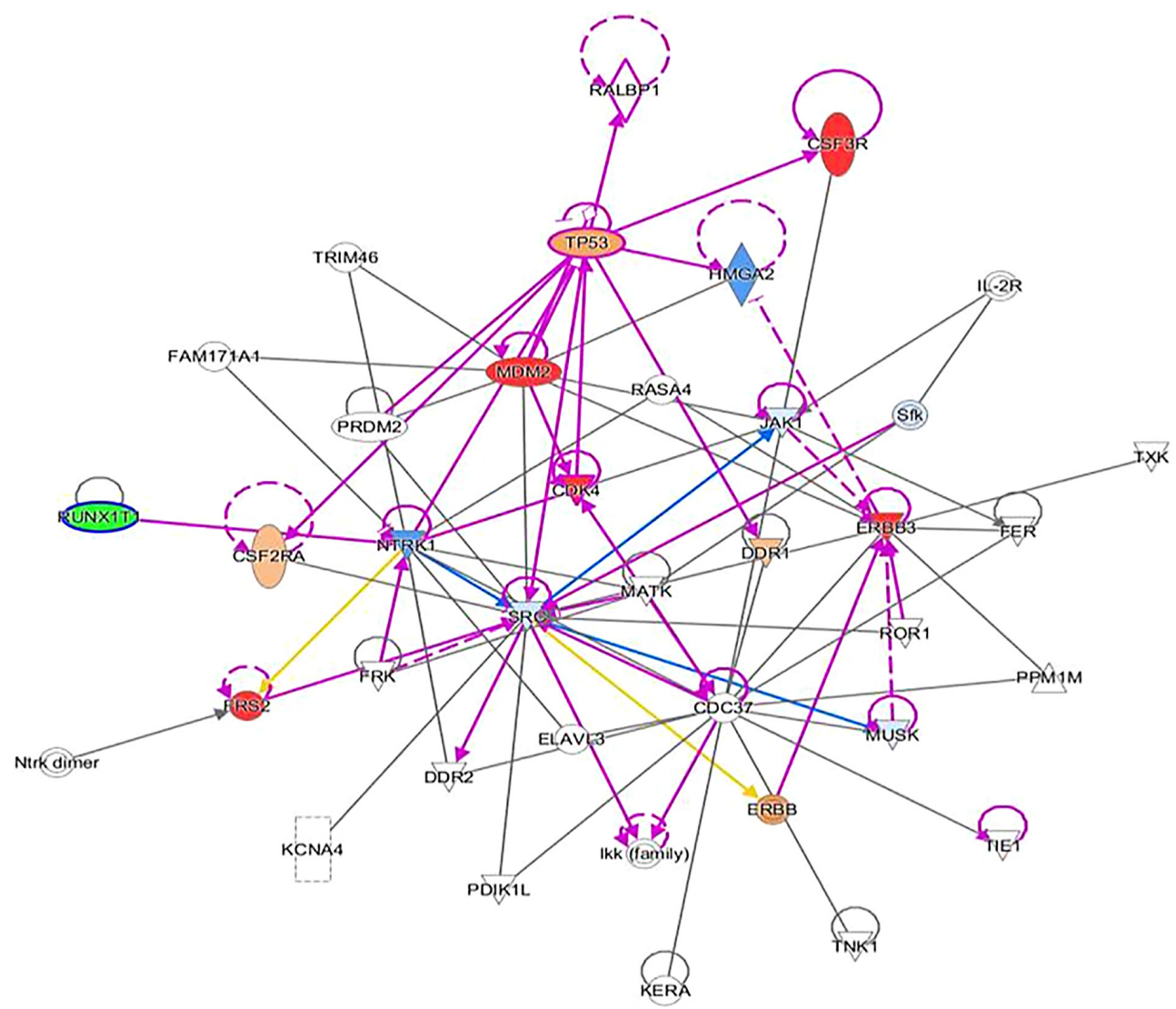

Figure 7. Ingenuity pathway analysis indicating RUNX1 inhibitors. Refer to Figure 4 legend for explanation of figure contents RUNX1, RUNX family transcription factor 1. 


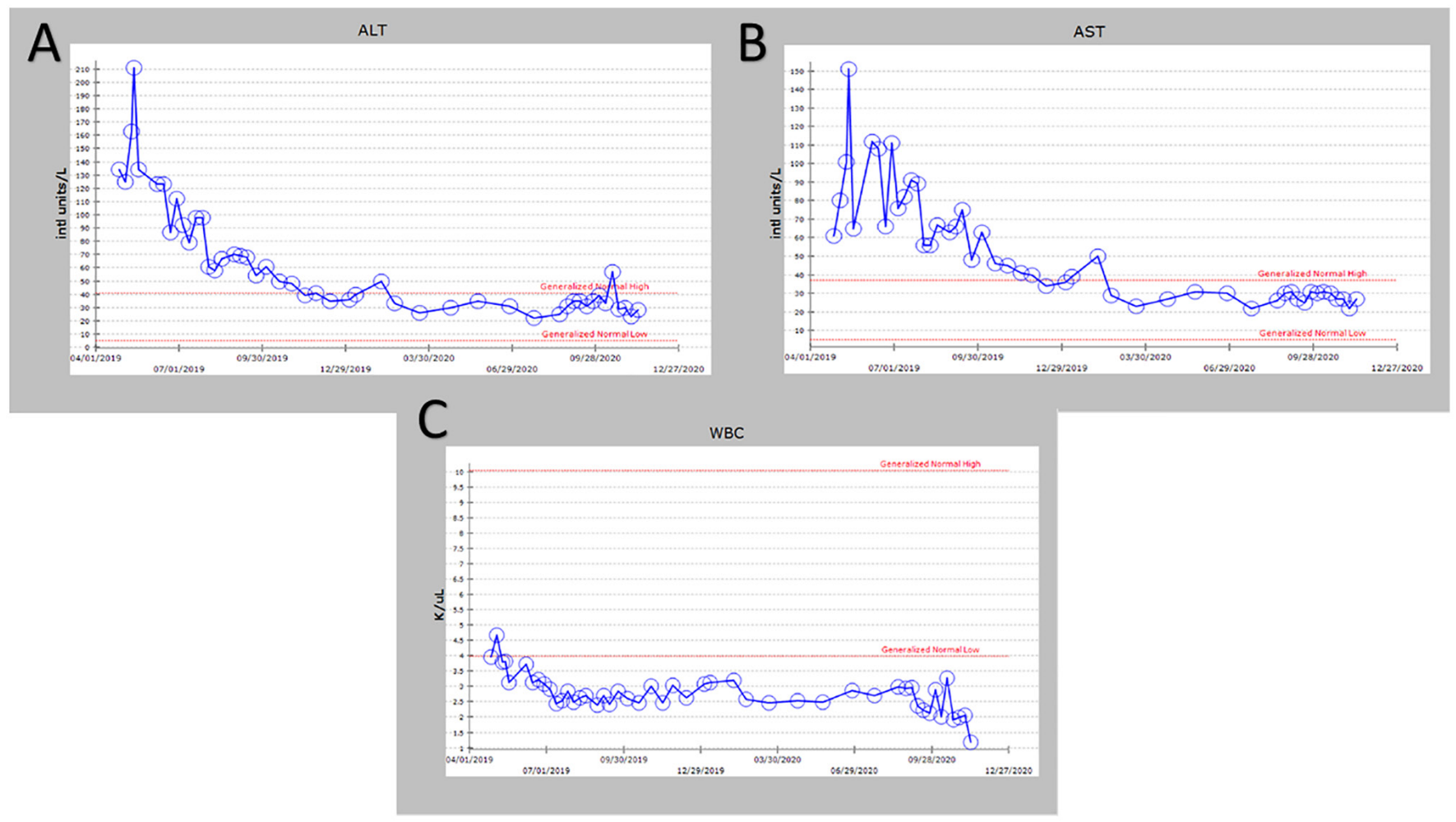

Figure 8. (A) ALT levels during pazopanib treatment; (B) AST levels during pazopanib treatment; (C) WBC levels during pazopanib treatment. WBC, white blood cells; ALT, alanine aminotransferase; AST, aspartate aminotransferase.

actions of SRC proto-oncogene and megakaryocyte-associated tyrosine kinase. According to the network analysis and activity predictors of mutated proteins (i.e. whether a given mutation increases or decreases the activity of a protein), the patient was subsequently prescribed $800 \mathrm{mg} /$ day of pazopanib, which is a tyrosine kinase inhibitor for the treatment for advanced/metastatic renal cell carcinoma and advanced soft tissue sarcomas. However, the patient developed elevated liver enzymes (alanine aminotransferase and aspartate aminotransferase >200 U/1) and mild hepatomegaly as indicated by abdominal ultrasound. The course of liver enzymes and white blood cell counts are presented in Fig. 8. Pazopanib was subsequently titrated down to $200 \mathrm{mg} /$ day, which reverted the liver enzyme levels back to normal levels. During the course of chemotherapy, the patient lost a total of 25 pounds in total body weight. The neck mass continued to reduce in size. The patient denied any changes in appetite, dysphagia or hoarseness. The dose of pazopanib was subsequently increased to $800 \mathrm{mg}$ /day without any further complications. The mass continued to decrease in size. Since the surgery two years ago and one year since chemotherapy ended, the patient is continuing to do well without any further growth of the primary tumor.

\section{Discussion}

AF is a benign, mesenchymal lesion composed of a proliferation of well-differentiated fibroblasts (1-4). The majority (two-thirds) of AF cases develop in the abdomen, while the remainder are found extra-abdominally (3). Specifically, $19-49 \%$ of AF cases are preceded by nonsurgical or surgical trauma. It is hypothesized that the pathogenesis involves an abnormal healing response with proliferation of immature fibroblasts, leading to a fibromatosis tumor (3). Aggressive fibromatosis of the head and neck regions are more aggressive variants of fibromatosis; they tend to be locally aggressive and have been known to erode bone, soft tissues and vital structures (3). As observed in the patient of the present study, clinical features, such as paraesthesia and weakness of the voice and hoarseness, occur because of pressure effects on local nerves and vascular structures (3). After treatment, these tumors have a high tendency to recur (40-70\%) with most cases recurring within 18 months of surgical excision (3), which was also observed in the present case. At present, the literature available to guide the treatment of wide local invasive AF is sparse. Treatment modalities include surgery, chemotherapy, radiotherapy, hormonal therapy or combinations thereof (1). The choice of treatment modality predominantly depends on the tumor location, tumor size, age of the patient and tumor profile (5). Given that the patient's AF had multiple mutations of unknown significance, the choice of the best initial chemotherapy agent for treating the AF was not obvious. Therefore, an IPA analysis was performed to determine potential chemotherapy agents that may be most effective. For the analysis, all amplification mutations were assumed to be gain-of-function mutations. In the IPA network for this patient's AF, the SRC protein was the central node for all the proteins and transcriptions factor interactions. Among the chemotherapy agents tested (pazopanib, ERBB, CDK4 and RUNX1 inhibitors), pazopanib was the only chemotherapy agent that was predicted to directly inhibit the activity of SRC. Pazopanib was started and dosed intermittently, and dosing was paused or reduced upon worsening of liver enzymes or the white 
blood cell count. The maximum response was seen within 6 months and subsequently, stable thickening of the neck persisted due to both residual disease and scarring resulting from treatment. At present, the patient remains on $800 \mathrm{mg}$ of pazopanib and is stable. If the tumor continues to recur after treatment with pazopanib, other chemotherapeutic agents will be screened using IPA analysis or repeat biopsies to interfere with tumor recurrence. At present, the standard treatment for head and neck AF is primary surgical excision with clear margins to minimize the chance of recurrence. However, the recurrence rate of AF is independent of the surgical margin status (1). Positive margins do not affect the overall survival rate or 5-year disease-free survival, making standard treatment with clear margins controversial (1). The few cases of AF that involve the larynx have been treated using a total or hemi-laryngectomy (2). The majority of reported cases $(66 \%)$ treated by hemi-laryngectomy and other less radical surgical interventions experienced recurrence of the primary tumor; of those that recurred in the larynx, $75 \%$ were treated by laryngectomy (2). Other treatment modalities, such as radiotherapy, appear to reduce local recurrence rates of AF in adults. However, $40 \%$ of patients suffered from severe complications, including pathologic fracture, pain, contracture, impaired range of motion and skin cancer (1). Chemotherapy regimens, including Vinblastine and Methotrexate, have lowered recurrence rates in pediatric patients (1). The decision regarding treatment regimens for patients with AF of the head and neck is best made by a multidisciplinary team, consisting of otolaryngologists, radiation oncologists and medical oncologists. These multidisciplinary teams have been indicated to improve decision-making and reduce waiting times for cancer patients (6). Regardless of the treatment modality applied, close follow-up and high suspicion of recurrence are necessary.

Controversy over treatment and management of AF in the head and neck region still exists. A minimal amount of literature on the topic is currently available, with even less available to guide the cases that involve the larynx. Case reports in the literature agree that surgery is the best treatment option but a consensus regarding the management of tumor recurrence has not been reached. Treatment and management, due to the lack of evidence in the literature, should be tailored to each individual patient and education of the risks and benefits of treatment modalities should be emphasized, since no treatment, even surgery with clear margins, guarantees prevention of recurrence. The use of multidisciplinary teams may improve decision making for these patients. The treatment of AF in the head and neck region is a topic that requires more attention and research.In the present case report, it was assumed that amplification mutations activated the proteins or transcription factors; further scientific analysis would be required to confirm this assumption. In general, sarcomas have more copy number alterations (amplifications, deletions, translocations) than point mutations, which makes choosing therapeutic targets difficult. Furthermore, numerous copy number alterations are unknown with respect to their effect on activating or deactivating proteins involved with critical functions in cellular metabolism, growth or cell cycle control $(7,8)$. Therefore, the use of IPA may provide an alternative method for determining chemotherapy agents to use for the treatment of difficult or rare tumors with genetic variants of unknown significance. In the present study, pazopanib was determined as an alternative chemotherapy regimen using the IPA analysis software and applied for treating the patient.

Administration of pazopanib is contraindicated for patients with severe hepatic impairment due to reports of severe or fatal hepatoxicity in clinical trials (9). The Food and Drug Administration (FDA) recommends monitoring hepatic function and to pause, reduce or discontinue dosing as indicated (9). Specifically, upon any indication of hepatotoxicity, the dose should be reduced to $200 \mathrm{mg} / \mathrm{day}$. The patient of the present study did not have any hepatic impairment. The FDA recommendations were followed and the dose of pazopanib was reduced to determine whether the increase in liver function tests (LFTs) was due to tumor breakdown or hepatic impairment. As was demonstrated, the patient's LFTs did not increase after the dose of pazopanib was increased to $800 \mathrm{mg} /$ day. Therefore, caution should be taken to completely withdraw a patient from pazopanib prior to reducing the dose and increasing it again to determine whether LFTs increase.

The current case report presented a rare and difficult to treat AF tumor for which therapeutic management has not been established. It was also demonstrated that even large tumors may be resected and there is a benefit even if a positive margin recurs through tumor burden reduction. Through the interactive network from IPA, pazopanib was theoretically predicted to be a potential treatment for this patient's AF. Pazopanib or Votrient is an FDA-approved tyrosine kinase inhibitor used in patients with advanced renal cell carcinoma or advanced soft tissue sarcoma who have received prior chemotherapy (9). Specifically, pazopanib is a multi-tyrosine kinase inhibitor of vascular endothelial growth factor receptor-1, -2 and -3 , platelet-derived growth factor receptor- $\alpha$ and $-\beta$, fibroblast grow th factor receptor- 1 and -3 , cytokine receptor, interleukin-2 receptor inducible T-cell kinase, leukocyte-specific protein tyrosine kinase and transmembrane glycoprotein receptor tyrosine kinase (9). Pazopanib is given once daily at 200 or $800 \mathrm{mg}$ /day depending on whether the patient has moderate renal or hepatic impairment. As demonstrated in the present case report, pazopanib may be an effective chemotherapy regimen in the treatment of aggressive AF.

\section{Acknowledgements}

Not applicable.

\section{Funding}

No funding was obtained.

\section{Availability of data and materials}

The data that support the findings of this study are available from the corresponding author upon reasonable request.

\section{Authors' contributions}

NL wrote the initial draft of the manuscript and was involved in the initial conception of the case report. JK contributed to the conception and the design of the study and contributed to the acquisition, analysis and interpretation of the data. SA edited 
the manuscript and performed the IPA analysis. JC reviewed the study for important intellectual content. All authors read and approved the final manuscript. JC and SA checked and approved the authenticity of the clinical and raw data used for the IPA analysis.

\section{Ethics approval and consent to participate}

Not applicable.

\section{Patient consent for publication}

The patient provided written informed consent for the publication of their data and images in this case report.

\section{Competing interests}

The authors declare that they have no competing interests.

\section{References}

1. Wang W, Koirala U, Ma S, Liu G, Ding M, Hu X and Lei D: Age-based treatment of aggressive fibromatosis in the head and neck region. J Oral Maxillofac Surg 72: 311-321, 2014.

2. Shinohara S, Suehiro A, Kikuchi M, Harada H, Kishimoto I and Imai Y: A case of desmoid tumor co-existing with recurrent squamous cell carcinoma in the larynx. Auris Nasus Larynx 44: 365-369, 2017.
3. Prabhu R, Natarajan A, Shenoy R and Vaidya K: Aggressive fibromatosis (desmoid tumour) of the head and neck: A benign neoplasm with high recurrence. BMJ Case Rep 2013: bcr2013200156, 2013.

4. Simões-Pereira J, Cabrera RA and Leite V: A case of thyroid fibromatosis, a rare lesion of this gland. Endocrinol Diabetes Metab Case Rep 2016: 16-0019, 2016.

5. Salas S, Dufresne A, Bui B, Blay JY, Terrier P, Ranchere-Vince D, Bonvalot S, Stoeckle E, Guillou L, Le Cesne A, et al: Prognostic factors influencing progression-free survival determined from a series of sporadic desmoid tumors: A wait-and-see policy according to tumor presentation. J Clin Oncol 29: 3553-3558, 2011.

6. Specchia ML, Frisicale EM, Carini E, Di Pilla A, Cappa D, Barbara A, Ricciardi W and Damiani G: The impact of tumor board on cancer care: Evidence from an umbrella review. BMC Health Serv Res 20: 73, 2020.

7. Cheng L, Pandya PH, Liu E, Chandra P, Wang L, Murray ME, Carter J, Ferguson M, Saadatzadeh MR, Bijangi-Visheshsaraei K, et al: Integration of genomic copy number variations and chemotherapy-response biomarkers in pediatric sarcoma. BMC Med Genomics 12 (Suppl 1): 23, 2019.

8. Cancer Genome Atlas Research Network: Electronic address: elizabeth.demicco@sinaihealthsystem.ca and Cancer Genome Atlas Research Network: Comprehensive and integrated genomic characterization of adult soft tissue sarcomas. Cell 171: 950-965, 2017.

9. Highlights of Prescribing Information - Votrient (pazopanib) tablets (2009). White Oak, Maryland: Food and Drug Administration. https://www.accessdata.fda.gov/drugsatfda_docs/ label/2015/022465s021lbl.pdf

(i) $($ This work is licensed under a Creative Commons Attribution-NonCommercial-NoDerivatives 4.0 International (CC BY-NC-ND 4.0) License. 